\title{
Paroles justes, paroles efficaces
}

Just Words, Right Words

\section{Gabrielle Radica}

\section{(2) OpenEdition}

Journals

Édition électronique

URL : http://journals.openedition.org/extremeorient/265

DOI : 10.4000/extremeorient.265

ISSN : 2108-7105

\section{Éditeur}

Presses universitaires de Vincennes

\section{Édition imprimée}

Date de publication : 1 novembre 2012

Pagination : 209-216

ISBN : 978-2-84292-352-5

ISSN : 0754-5010

\section{Référence électronique}

Gabrielle Radica, «Paroles justes, paroles efficaces », Extrême-Orient Extrême-Occident [En ligne], 34 | 2012, mis en ligne le 01 novembre 2015, consulté le 19 avril 2019. URL : http:// journals.openedition.org/extremeorient/265; DOI : 10.4000/extremeorient.265 


\title{
Paroles justes, paroles efficaces
}

\author{
Gabrielle Radica
}

En ce qu'elle concerne le pouvoir du langage, la rhétorique, ou art de parler et de persuader, est par elle-même une compétence politique. À ce titre, le prédicateur, l'avocat ou l'amoureux intrigant qui parviennent à modifier les dispositions et les comportements de leurs interlocuteurs en usant efficacement de leur parole pratiquent une sorte de politique entendue en un sens métaphorique. Mais la politique désigne plus spécifiquement une sphère d'activités et de rapports humains, celle de la distribution du pouvoir social, de la détermination de valeurs et de normes collectives, et de la prise de décisions qui engagent le destin d'une communauté, sphère dans laquelle la rhétorique est appelée à exercer un rôle plus précis. En ce second sens du terme «politique», la rhétorique des gouvernants, des conseillers, des idéologues et autres partisans, ou encore celle du simple citoyen se sépare de la rhétorique «de la chaire » et de celle «du barreau». Tel est le domaine des relations entre rhétorique et politique qui est examiné dans le présent volume.

Les situations rhétoriques qu'analysent les différentes contributions ne montrent pas de prise de parole devant une assemblée, ni nécessairement de délibération sur les fins collectives poursuivies par une communauté ${ }^{1}$, ni enfin d'échange égalitaire et démocratique, toutes conditions que l'on pourrait croire aujourd'hui essentielles à la nature du lien politique. Dans l'Essai sur l'origine des langues, au chapitre XX, Rousseau déploie sur le plan linguistique l'argument développé dans le Contrat social, selon lequel une association d'hommes mus par un intérêt commun est seule digne d'être appelée société. Tout autre lien entre les hommes court le risque de n'être qu'une agrégation d'hommes rassemblés par la force.

1. L'article de Yuri Pines sur Shang Yang présente à cet égard une exception. 
Dans les anciens temps où la persuasion tenait lieu de force publique, l'éloquence était nécessaire. À quoi servirait-elle aujourd'hui que la force publique supplée à la persuasion? L'on n'a besoin ni d'art ni de figure pour dire, tel est mon plaisir. Quels discours restent donc à faire au peuple assemblé ? Des sermons. Et qu'importe à ceux qui les font de persuader le peuple, puisque ce n'est pas lui qui nomme aux bénéfices?

S'appuyant sur l'hypothèse pessimiste d'une corruption inévitable des sociétés légitimes en sociétés illégitimes, l'auteur ajoute:

Les sociétés ont pris leur dernière forme; on n'y change plus rien qu'avec du canon et des écus, et comme on n'a plus rien à dire au peuple sinon, donnez de l'argent, on le dit avec des placards au coin des rues ou des soldats dans les maisons; il ne faut assembler personne pour cela; au contraire, il faut tenir les sujets épars, c'est la première maxime de la politique moderne ${ }^{2}$.

La société n'existe en effet chez cet auteur que fondée sur le contrat social, à savoir une parole échangée entre égaux, et reconduite dans son existence à chaque délibération portant sur l'intérêt collectif. Les rapports de domination et d'influence sont rejetés hors de la politique car ils courent le risque d'être mêlés de force et de violence illégitimes.

Pour autant, ces conditions de la parole politique sont fortement situées dans le temps et l'espace, à savoir dans l'horizon d'une tradition démocratique européenne qui prend pour modèles - souvent idéalisés ou reconstruits - la démocratie athénienne, les assemblées du sénat et du peuple à Rome, la pratique oratoire des parlements anglais, américain et français. Mais cette définition de la politique aboutit paradoxalement à supprimer le recours à la rhétorique: Rousseau condamne la démagogie et pense que l'on ne doit délibérer qu'en son for intérieur sur l'intérêt commun; la reconnaissance de ce dernier doit être l'objet d'une conviction et même d'une persuasion, dans laquelle toutefois nulle manipulation extérieure, nulle déformation partisane ne doivent interférer. Cette conception puriste de la politique ignore toutes les situations intermédiaires comme les entreprises diplomatiques, les négociations, les moments de crise et de redéfinition du lien politique, les situations de guerre où paroles et violences se trouvent imbriquées. Aussi, remettre en question ces réquisits sur la nature du lien politique permettrait peut-être de faire ressurgir le lieu des rapports entre rhétorique et politique comme celui précisément où l'intérêt général n'est pas opposé strictement aux intérêts privés et attend de recevoir sa définition de différents discours, comme celui où le règne de la force ne se distingue pas de celui du droit et du lien pacifique, comme celui où l'inégalité de statut est

2. Rousseau, Jean-Jacques (1959-1995). CEuvres complètes. 5 vol., éd. Gagnebin et Raymond. Paris, Gallimard, «Bibliothèque de la Pléiade»: t. 5, 428. 
parfois renversée par une inégalité oratoire symétrique. En d'autres termes, c'est moins en lisant Platon - selon qui le sophiste s'oppose au philosophe-roi, et pour qui la cité doit se régler sur le Vrai - ou Rousseau, lequel coupe strictement le droit du domaine de la violence, ou encore, plus près de nous, Ricœur, qui affirme que la parole politique doit échapper au piège de la «manipulation fallacieuse $^{3}$ », qu'en lisant Aristote ou Tocqueville, chez qui les intérêts se composent et se définissent par négociation, discussion et compromis, ou encore Naudé, Machiavel et les penseurs de la raison d'État en général, qui font de la politique l'objet des arcana imperii, que l'on pourra comprendre les rapports de la rhétorique et de la politique. Au lieu d'opposer une tradition européenne à une tradition chinoise concernant la pensée de ces rapports entre rhétorique et politique, il conviendrait plutôt d'opposer les conceptions qui ignorent leur interaction à celles qui la précisent et l'élaborent, puisque cette opposition apparaît aussi bien dans le contexte chinois: Confucius distingue entre une éthique de la parole exigeante, où les mots devraient se raréfier et correspondre le mieux possible aux actions d'une part, et l'accommodement nécessaire avec les circonstances qui contraint d'accepter l'avènement des orateurs politiques d'autre part (voir l'article d'Albert Galvany, «Sly mouths and silver tongues »).

Les articles présentés dans le volume définissent des rapports entre rhétorique et politique où la parole doit engager ou arrêter la violence militaire, où l'intérêt particulier du conseiller s'associe à l'intérêt collectif, converge avec celui du souverain ou au contraire s'en écarte, où le lien politique ne repose pas sur une parole performative ressaisissant et refondant à chaque fois la totalité de la société, mais se tisse dans une série de décisions et d'actes particuliers, influencés par les discours, et capables ou non de produire et maintenir un État prospère.

Or, dès lors que la parole est étudiée moins dans ses rapports à la vérité qu'en tant qu'action, ce sont les conditions et les circonstances dans lesquelles cette action prend place qui importent pour l'analyse : la lecture des articles de ce volume permet de développer plus particulièrement trois remarques sur les rapports qu'entretiennent la rhétorique et la politique, liées à la singularité du contexte chinois. La première concerne la détermination qu'impose la forme du gouvernement et des institutions aux modalités de la parole; la seconde, la façon dont le danger de parler et celui de se laisser persuader impriment au discours des caractéristiques particulières qu'il est au demeurant délicat de reconstituer (comment parler à demi-mot ou écrire entre les lignes ?) ; enfin, la dernière remarque porte sur la difficulté de mettre cette parole en règles, et de considérer la compétence rhétorique comme un art.

3. Ricœur, Paul (1991). «Langage politique et rhétorique». In Ricœur, Paul, Lectures 1. Autour du politique. Paris, Éditions du Seuil : 166. 


\section{Les conditions institutionnelles d'exercice de la rhétorique}

Le déploiement de l'art oratoire dépend fortement des conditions politiques qui lui sont offertes pour s'exercer: si les hommes du XVIII ${ }^{e}$ siècle ne pensaient plus voir réapparaître les conditions grecques ou romaines de la vraie éloquence, ils faisaient la différence entre le régime anglais parlementaire qui revivifie la possibilité de l'art oratoire d'une part, et le régime monarchique français dans lequel seule la chaire donne carrière à l'éloquence. On n'est pas éloquent à propos d'un mur mitoyen affirme Voltaire dans l'article «Éloquence» de l'Encyclopédie, mais seulement sur des intérêts collectifs qui emportent une adhésion large. On l'a vu, le peuple assemblé, la liberté de parole, l'importance de la discussion et de l'égalité des participants semblent propices au développement de l'éloquence. Pourtant la relation monarchique ou impériale fortement inégalitaire n'aura pas empêché, ainsi que le montrent les articles de ce volume, le déploiement d'une rhétorique particulière, celle de ces conseillers voyageurs doués dans l'art de la parole, qui adressaient aux différents souverains des discours visant à influencer leurs décisions.

De telles conditions politiques influent sur la nature de cette rhétorique. En premier lieu, le contexte géopolitique, une situation de redéfinition permanente des frontières et d'instabilité militaire orientent, le regard sur l'efficacité du discours et font de la sanction des événements la sanction du discours de l'orateur. En second lieu, la nature des relations de l'orateur et du souverain s'avère également déterminante. Dans L'Homme de cour, Baltasar Gracian (1601-1658) énonce les conditions d'une maîtrise de soi et d'une vie réussie dans une société curiale, reposant sur un système de distinctions, mais aussi sur différents commerces, échanges de services et de faveurs, ainsi que sur l'importance de la réputation. L'art de converser et de négocier est essentiel, et situé en ordre d'importance immédiatement en dessous de l'action. Aussi l'homme habile s'emploie-t-il à faire avancer ses objectifs en étudiant son adversaire et en le trompant éventuellement sur ses fins: il peut par exemple essayer de lui faire croire qu'il épouse sa cause, lui mentir, etc. Mais le jeu se complexifie, car l'interlocuteur se prémunit à son tour contre de telles tromperies, engageant l'adversaire à se prémunir derechef, etc. Il arrive que l'homme habile redouble de tromperie en ne trompant plus, et en «usant» de la vérité comme d'un instrument de tromperie. Le jeu d'entre-ajustement ne s'arrête pas tant que l'échange continue et que les paroles circulent dans un sens et dans l'autre. Rien de tel qui soit concevable dans les situations typiques décrites dans les contributions du présent numéro, dans la mesure où la complexification indéfinie des règles du jeu subit un coup d'arrêt inévitable en raison de l'asymétrie absolue qui caractérise les rapports du conseiller et du souverain. Celle-ci interdit un tel processus de raffinement prolongé des procédés rhétoriques et des manipulations. 
L'orateur-écrivain se trouve dans une situation radicalement différente de celle du souverain, qui, pour sa part, répond à l'éventuelle tentative de manipulation de l'orateur en lui opposant une apparence impénétrable, préludant parfois à une sanction fatale en cas d'échec de l'action recommandée, d'insatisfaction personnelle, ou simplement d'inconvenance jugée intolérable du propos tenu par le conseiller. Idéalement, le souverain ne parle pas, ou seulement pour juger et trancher; par-dessus tout, il évite d'entrer dans le jeu des échanges oratoires ou des joutes verbales (voir la première partie de l'article de Romain Graziani sur le Han Feizi).

\section{Rhétorique et persécution}

L'orateur risque beaucoup dans ces discours et il développe de ce fait un art de s'exprimer qui doit non seulement atteindre les objectifs de persuasion, mais aussi garantir sa survie : connaissant les risques, certains orateurs commencent leur discours par des appels à la sanction, peut-être pour mieux les conjurer ${ }^{4}$.

La censure et le danger de parler, accrus par le contexte politique inégalitaire, n'empêchent pourtant pas de faire passer un message; ces entreprises renvoient à la question traitée par Leo Strauss de l'art d'écrire en contexte de persécution, ou art d' «écrire entre les lignes ${ }^{5} »$. «La persécution [...] ne peut empêcher la pensée indépendante. Elle ne peut même pas en empêcher l'expression », affirme Strauss. «La persécution ne peut même pas empêcher l'expression publique de la vérité hétérodoxe, car un homme dont la pensée est indépendante peut exprimer publiquement ses opinions sans dommage, pourvu qu'il agisse avec prudence. Il peut même les faire imprimer, sans courir aucun danger, pourvu qu'il soit capable d'écrire entre les lignes» (p. 56-57). Défendre une telle hypothèse à propos de l'écrit suppose d'y voir à l'œuvre une «technique particulière d'écriture» qui contraint au demeurant à une technique particulière de lecture toujours soumise à révision. L'art d'écrire est un art paradoxal qui peut se définir de différentes façons : faire passer un message à certains et pas à d'autres dans le même texte, ou bien un message à certains et un message différent à d'autres.

Certes, Strauss s'intéresse à des situations où une censure établie ou bien une condamnation sociale plus diffuse guettent l'écrivain qui s'écarte de l'orthodoxie, et il privilégie le cas des philosophes à l'esprit indépendant

4. Ce péril propre à la situation de conseiller n'est pas lié au contexte autoritaire et hiérarchique, puisque l'on doit rappeler que les Spartiates proposaient des lois la corde au cou. Toutefois les dangers qui guettent celui qui s'est exprimé imprudemment semblent omniprésents dans les descriptions offertes par l'ensemble des articles ici recueillis.

5. Strauss, Leo (1989). «La persécution et l'art d'écrire ». In Strauss, Leo, La Persécution et l'Art d'écrire, trad. Olivier Berrichon-Sedeyn. Paris, Press Pocket: 55-74. 
qui s'émancipent de la pensée dominante, alors que les écrivains et historiens dont il est question ici craignent plus immédiatement la sanction redoutable du souverain. Mais il n'en demeure pas moins que c'est bien un tel art qui est décrit dans les troisième, quatrième et cinquième articles de ce recueil: ne pourrait-on par exemple supposer un double lectorat auquel s'adresserait simultanément Shang Yang (p. 18), non pas, comme c'est le cas chez Strauss, d'une part l'élite éclairée, d'autre part le vulgaire, mais d'une part les élites en place et d'autre part, une frange marginalisée de la population aspirant à des postes de pouvoir? (Pour ce qui est de l'utilisation de l'expression fourre-tout de «min ben», «le peuple au fondement de la pratique politique», par le conseiller de la maison des Han, Jia Yi, il est bien question en revanche de repérer simultanément un message élitiste et un message adressé au peuple, grâce à l'ambivalence de la formule, qui n'est en rien précurseur du concept de démocratie d'après l'auteur de l'article.)

C'est encore un art d'écrire raffiné qu'expose Dorothee Schaab-Hanke à propos des historiens père et fils Sima Tan et Sima Qian qui, dans les Mémoires historiques, réussissent à pratiquer une «rhétorique de trahison» envers l'empereur Wu par la référence à un passage du Gongyang Zhuan relatant le moment où Confucius perd l'espoir de voir apparaître un sage gouvernant, à cause du mauvais augure que représente à ses yeux la capture d'une licorne, et où il n'envisage plus sa venue que dans un futur éloigné. Or la référence à ce passage est une occasion dans les Mémoires historiques de critiquer l'expansionnisme de l'empereur $\mathrm{Wu}$, sa gestion arbitraire des fiefs, ses naïvetés et inconvenances dans l'usage des sacrifices. Aussi la compréhension de ces ouvrages requiert-elle un art de lire qui n'est jamais assuré d'atteindre la certitude, mais doit risquer des hypothèses plausibles afin de distinguer la part plus conciliatrice du père, la part cynique et désabusée du fils. À cet égard, la différence entre l'histoire et la fiction apparaît des plus ténues : l'article de Paul van Els montre à partir de cas précis combien l'étude minutieuse du recours aux anecdotes peut être féconde et révéler que telle ou telle façon de raconter la même anecdote peut promouvoir une vision politique du monde contre une autre.

\section{Rhétorique, art militaire et médecine}

Concevoir la rhétorique comme un art présente quelques paradoxes, ce que montrent Shang Yang, qui recourt à l'écriture pour dénoncer les arts, et l'exaspérant charron qui dénigre les lectures du duc Huan (Paul van Els, «Tilting Vessels and Collapsing Walls »), pointant ainsi tous deux, l'un comme auteur, l'autre comme personnage, le problème de l'art rhétorique. Leur attitude rappelle celle de Rousseau condamnant les arts tout en y voyant le dernier remède à la 
corruption qu'ils ont causée, ou encore celle de Socrate qui affiche son mépris pour les œuvres écrites. L'art rhétorique est en effet condamné à user de paroles pour provoquer un changement à un niveau sur lequel les paroles n'ont pas de prise certaine, ou qui relève d'un autre domaine qu'elles: les motifs, les intentions. D'où le caractère toujours insuffisant de la parole, et le risque de fonctionner à vide sans s'arrimer aux choses sur lesquelles elle cherche pourtant à opérer. Quoique dans sa visée même la rhétorique soit par essence compromise, les pages livrées ici permettent d'en éclairer le statut technique.

Si l'on considère l'ordre des moyens et des fins, ainsi que l'architectonique des arts, on constate que la rhétorique prélude ou succède au recours à l'art militaire (on incite à poursuivre une guerre, on en dissuade), qu'elle collabore avec lui, voire qu'elle y supplée (les paroles justes de Xiangzi, étudiées par Van Els, sur le rempart effondré lui valent une victoire sur le peuple mieux remportée que par n'importe quelle manœuvre militaire). Mais si l'on considère en quoi consiste l'activité, une analogie de structure est également repérable avec l'art militaire, puisque l'orateur est une sorte de stratège qui prend possession de l'âme de son adversaire. Ceci révèle autant d'après Albert Galvany la violence à l'œuvre dans l'art oratoire que l'intelligence et le savoir déployés dans toute stratégie: la connaissance de l'adversaire (le souverain) devra être bien plus précise et singulière que les opinions que le rhéteur grec prête grosso modo à l'assemblée qu'il veut convaincre. Le registre de la neutralité et de la transparence porte des connotations très différentes chez un auteur comme Platon et dans le contexte chinois. Alors que le sophiste est présenté dans La République comme celui qui promène un miroir sur la foule et ne fait que lui renvoyer ce qu'elle est, l'orateur chinois doit imiter le stratège militaire, en devenant aussi fluide que l'eau pour échapper à la saisie de l'adversaire.

Savoir s'adapter à toute situation et se rendre insaisissable à force de changer, c'est développer un savoir pratique et singulier, loin des recettes et des mauvaises généralisations auxquelles se réduit l'art sophistique selon Platon ${ }^{6}$. L'emploi de l'art oratoire est paradoxal au sens où sa victoire comme sa défaite le disqualifient semblablement: en cas de défaite, la sanction de l'événement retombe sur les conseils du mauvais orateur et en cas de victoire, il faut comprendre qu'on l'avait obtenue avant même de parler, parce qu'on a suffisamment miré l'adversaire (voir l'article de Galvany).

La rhétorique requiert donc un savoir psychologique. Si elle peut être tantôt poison, tantôt remède, ce n'est pas parce qu'elle dirait tantôt la vérité, tantôt la déformerait. Romain Graziani montre à cet égard que la rhétorique peut soigner selon des voies dont on ne peut totalement rendre compte, et devient même une sorte de travail «psychanalytique» avant la lettre.

6. Voir le Protagoras et le Phèdre. 
